\title{
Theoretical Study of the Mean and Resultant Change of Interaction Frequency for Oxygen-Oxygen Ion Dipoles in $\mathrm{CaF}_{2}$ Crystal
}

\author{
Farouk A. Kasir \\ Department of Physics \\ Mohanad M. Alyas \\ College of Science \\ University of Mosul
}

E-mail: $\underline{\text { mohanad.moaid@yahoo.com }}$

(Received 20/6/2012 ; Accepted 8/10/2012)

\begin{abstract}
The purpose of this paper is to analyze and study the angular variation, in the presence of the external magnetic field, of the mean and resultant displacements of the dipole-dipole interaction frequency, $\mathrm{O}^{-}-\mathrm{O}^{-}$ion dipoles in $\mathrm{CaF}_{2}$ crystal. The dipolar interaction frequencies of six sites of equivalent ions are described by their geometrical parts of their tensors and analyzed for four different cases. The conclusion is that the theoretical method provides a satisfactory approach in cases for which there are adequate data.
\end{abstract}

Keywords: Interaction frequency, ion dipoles, $\mathrm{CaF}_{2}$ crystal.

\section{درلسة قارية لمطل ومحصاة الثغييرفي تردد الثفل المتبادلة الثائي النماب من نوع أوكسجين -أوكسجين في بلورةفلوربد الكالسيو}

\section{المالغ}

أن الهذف من هذا العمل هو، إجراء درلسة ظارية وتحليلية للتردد الناتج عن الأفعل المتبادلة مابين

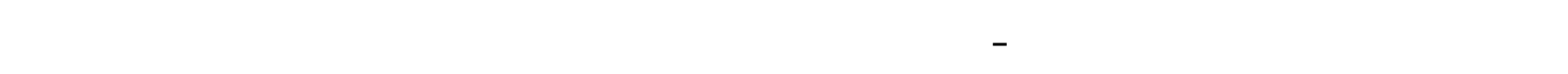

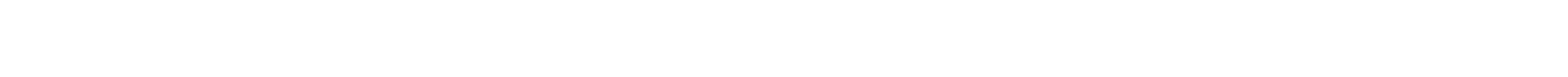

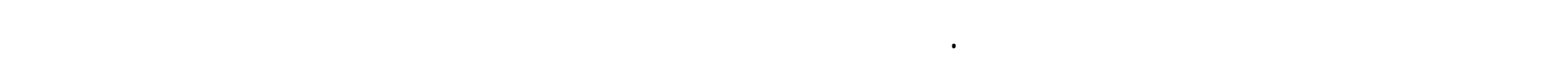

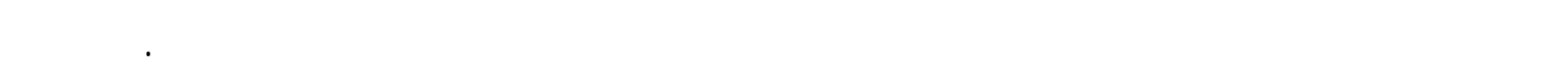

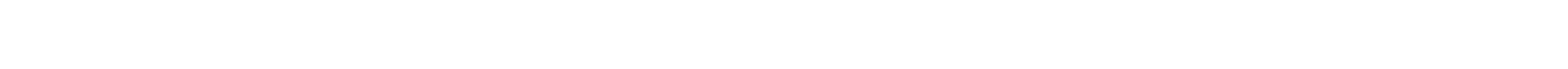
المقترح.

\section{INTRODUCTION}

Many studies involving experimental techniques have been done in an attempt to understand the behavior of oxygen centers in $\mathrm{CaF}_{2}$ crystals. Calcium fluoride is a suitable material for a variety laser optical applications in the ultraviolet spectral region. It can be 
used for generating high-order harmonics and white light from ultra short laser pulses (Megerle et al., 2009). On the other hand, one of the big unsolved problems in the application of $\mathrm{CaF}_{2}$ as an optical material is the oxygen contamination in the bulk and at the surface. Oxygen is readily incorporated into the bulk during crystal growth. For this reason, we say that one of the main obstacles to exploiting $\mathrm{CaF}_{2}$ is the presence of oxygen centers, having an optical absorption in the UV region (Sils et al., 2007). Therefore, a comprehensive effort is undertaken for calculating geometrical structures and optical properties of oxygen centers and related defects in $\mathrm{CaF}_{2}$.

In growing process, substitution oxide ion impurities are compensated by anion vacancies $\mathrm{V}_{\mathrm{A}}$ and form $\mathrm{O}^{-2}-\mathrm{V}_{\mathrm{A}}$ dipoles. The properties of this dipole have been studied experimentally. However, accurate theoretical modeling of the atomic scale mechanisms in the $\mathrm{O}^{-2}-\mathrm{V}_{\mathrm{A}}$ dipole is still lacking (Mysovsky et al., 2005). As a result of the photo dissociation when the temperature exceeds $200^{\circ} \mathrm{K}$, the concentration of the $\mathrm{O}^{-2}-\mathrm{V}_{\mathrm{A}}$ dipoles decreases and other defect species, such as isolated substitutional $\mathrm{O}^{-}$ions and complexes of $\mathrm{O}^{-2}$ with neutral and charged anion vacancies are observed. The $\mathrm{O}^{-}$impurities formed during photo dissociation, were studied experimentally by means of EPR spectroscopy, which showed no optical absorption associated with $\mathrm{O}^{-}$ions (Radzhbov et al.,1986).

From a theoretical point of view, modeling of anion vacancies and oxygen impurities face several problems, because accurate modeling of the magnetic properties of these defects requires describing a large number of atoms near them quantum mechanically. In addition, vacancies and oxygen impurities aggregate into complex defects which induce atomic displacements in several tens of atoms near the defect site. For example, to clarify the electron structure and atomic geometry of oxygen-vacancy dipoles, needed to be taken into account where the supercell containing 96 atoms (Shi et al., 2007).

The purpose of this paper is to make an accurate calculation of oxygen-oxygen ions defect in the bulk $\mathrm{CaF}_{2}$ and to predict their spectroscopic properties. Detailed quantitative calculations have been performed to analyze the angular dependence of the mean and resultant change of interaction frequency for oxygen-oxygen dipole center in the presence of an external magnetic field.

\section{THEORETICAL PROCEDURE}

In an ordinary paramagnetic crystal, the nature of the mutual interactions between paramagnetic ions and their effects on the paramagnetic resonance frequency are considered in this work.

The most obvious interaction between these ions is dipole-dipole interaction, which arises from the influence of the magnetic field of one ion on the dipole moments of neighbouring ions. Furthermore if an external magnetic field acts on the crystal, the local field at each ion must be added in a vector form to it. If the local field is small compared with the external magnetic field, only the component of the former parallel to the latter is important and this component varies from site to site, giving a displacement to the resonance interaction frequency of each ion (Abragam et al., 1970). 
From classical theory, the energy of two point magnetic dipoles $\overrightarrow{\mathbf{m}}_{i}$ and $\overrightarrow{\mathbf{m}}_{\mathrm{j}}$, a distance r a part, is:

$$
W=\mathbf{r}^{-3}\left\{\overrightarrow{\mathbf{m}}_{\mathrm{i}} \cdot \overrightarrow{\mathbf{m}}_{\mathrm{j}}-3 \mathbf{r}^{-2}\left(\overrightarrow{\mathbf{m}}_{\mathrm{i}} \cdot \overrightarrow{\mathbf{r}}\right)\left(\overrightarrow{\mathbf{m}}_{\mathrm{j}} \cdot \overrightarrow{\mathbf{r}}\right)\right\}
$$

For a pair of isotropic electronic dipoles of magnetic moments $\left(\overrightarrow{\mathbf{m}}_{\mathrm{i}}=-\mathrm{g}_{\mathrm{i}} \beta \overrightarrow{\mathbf{S}}_{\mathrm{i}}\right)$ and $\left(\overrightarrow{\mathbf{m}}_{\mathrm{j}}=-\mathrm{g}_{\mathrm{j}} \beta \overrightarrow{\mathbf{S}}_{\mathrm{j}}\right)$, equation (1) becomes in operator form as

$$
\hat{\mathbf{H}}=\mathrm{g}_{\mathrm{i}} \mathrm{g}_{\mathrm{j}} \boldsymbol{\beta}^{2} \mathbf{r}^{-3}\left\{\overrightarrow{\mathbf{S}}_{\mathrm{i}} \cdot \overrightarrow{\mathbf{S}}_{\mathrm{j}}-3 \mathbf{r}^{-2}\left(\overrightarrow{\mathbf{S}}_{\mathrm{i}} \cdot \overrightarrow{\mathbf{r}}\right)\left(\overrightarrow{\mathbf{S}}_{\mathrm{j}} \cdot \overrightarrow{\mathbf{r}}\right)\right\}
$$

If the direction cosines of $\overrightarrow{\mathbf{r}}$ are (l, $\mathbf{m}, \mathbf{n})$, equation (2) can be expanded:

$$
\begin{aligned}
& \hat{\mathrm{H}}=\mathrm{g}_{\mathrm{i}} \mathrm{g}_{j} \beta^{2} \mathrm{r}^{-3}\left\{\mathrm{~S}_{\mathrm{ix}} \mathrm{S}_{\mathrm{jx}}\left(1-3 \mathrm{l}^{2}\right)+\mathrm{S}_{\mathrm{iy}} \mathrm{S}_{\mathrm{iy}}\left(1-3 \mathrm{~m}^{2}\right)+\right. \\
& s_{i z} s_{i z}\left(1-3 n^{2}\right)=\left(s_{i X} s_{i y}+s_{i y} s_{j X}\right) 3 l m-\left(s_{i y} s_{i z}+\right. \\
& \left.\left.\mathbf{S}_{\mathrm{iz}} \mathbf{S}_{\mathrm{iy}}\right) 3 \mathrm{mn}-\left(\mathrm{s}_{\mathrm{iz}} \mathrm{S}_{\mathrm{jx}}+\mathrm{S}_{\mathrm{ix}} \mathbf{S}_{\mathrm{j} z}\right) 3 \mathrm{nl}\right\}
\end{aligned}
$$

Which is a symmetric tensor type interaction.

Using the foregoing approach, an expression describing the dipolar interaction frequency $\mathbf{f}_{\mathrm{dd}}$ has been found by expanding the vector relationships for $\overrightarrow{\mathbf{s}}_{\mathrm{i}}, \overrightarrow{\mathbf{s}}_{\mathrm{i}}$ and $\overrightarrow{\mathbf{r}}$ and performing the indicated scalar products. The resulting expansion can be expressed in a compact notation as(Graybeal,1988)

$$
\mathrm{f}_{\mathrm{dd}}=\mathrm{g}_{\mathrm{i}} \mathrm{g}_{\mathrm{i}} \beta^{2}\left(\overrightarrow{\mathbf{s}}_{\mathrm{i}}, \underline{\mathrm{D}} \cdot \underline{\overrightarrow{\mathbf{s}}_{\mathrm{i}}}\right) / \mathrm{h}
$$

where $\underline{\mathbf{D}}$, is the dipole coupling tenser, and for the case of identical spins, $\mathbf{S}_{\mathrm{i}}=\mathbf{S}_{\mathbf{j}}=\mathbf{1} / \mathbf{2}$, the dipolar frequency tensor A will have the following form,

$$
\underline{A}=f_{\max }\left|\begin{array}{ccc}
31^{2}-1 & 3 \operatorname{lm} & 3 n l \\
3 \operatorname{lm} & 3 m^{2}-1 & 3 m n \\
3 n l & 3 m n & 3 n^{2}-1
\end{array}\right|
$$

where $(\mathbf{l}, \mathbf{m}, \mathbf{n})$ are the direction cosines of $\overrightarrow{\mathbf{r}}_{\mathbf{i j}}$ and $\mathbf{f}_{\max }$ is the normalized maximum dipolar frequency.

In the presence of an external magnetic field $\overline{\mathbf{B}}$ applied along an arbitrary axis, the resonance frequency of each ion is displaced due to the effect of the magnetic dipole-dipole interaction. In the high field approximation, the displacement in frequency can be represented in one dimension for a pair of like spins, i.e., one frequency component, by the following modified formula (Graybeal, 1988 ; Kasir, 1994). 


$$
\mathbf{f}=\gamma \cdot \overline{\mathrm{B}}-\left(\mathbf{A}_{\mathrm{xx}} \mathrm{M} \cos \phi+\mathrm{A}_{\mathrm{xy}} \mathrm{M} \cos \Psi+\mathbf{A}_{\mathrm{xz}} \mathrm{M} \cos \boldsymbol{\theta}\right)
$$

where each spin is quantized along the magnetic field $\overline{\mathbf{B}}, \mathbf{M}$ is the projection of the spin along the principle c-axis of the crystal, $\gamma$ the magnetogyric ratio and $(\phi, \Psi, \theta)$ are the angles between the magnetic field vector and the selected coordination system of axes $(\mathrm{x}, \mathrm{y}, \mathrm{z})$ respectively.

\section{CALCULATION AND RESULTS}

In this work, the model postulated for the location of the oxygen center in $\mathrm{CaF}_{2}$ is shown in Fig. (1), presumed that $\mathrm{O}^{-}$ions replace two adjacent fluorine ions (other possibilities may occur), and these types of defects may occur either naturally or produced by photo dissociation of other complex oxygen impurities.

Taking into account the nearest and next nearest neighbours, one may distinguish six different sites according to the coordinate system (xyz) assumed for the $\mathrm{O}^{-}-\mathrm{O}^{-}$center in Fig. (1). Each site represents a state of a group of equivalent ions.

Calculations have been done to determine the position vector for each individual ion taking into account that the distance between fluorine-fluorine ions parallel to [100] in $\mathrm{CaF}_{2}$ is $0.2725 \mathrm{~nm}$. The normalized maximum dipolar interaction frequencies were calculated using the following parameter values: Fluorine magnetogyric ratio=40.07 MHz/Tesla, $\mathbf{g}=2.01$, $\mathbf{S}=1 / 2$ and $\boldsymbol{\beta}=9.2732 \times 10^{-24}$ Joules/Tesla (Kasir, 1994).

The direction cosines of $\overrightarrow{\mathbf{r}}$ for each site, ( Fig. 2), were calculated Table (1) and then combined with the $\mathbf{f}_{\max }$, to get the geometric parts of tensors for each site which result from the main interaction axes. The values of the normalized interaction frequency and the geometric parts of tensors obtained are shown in (Table 2), for the six different sites. 


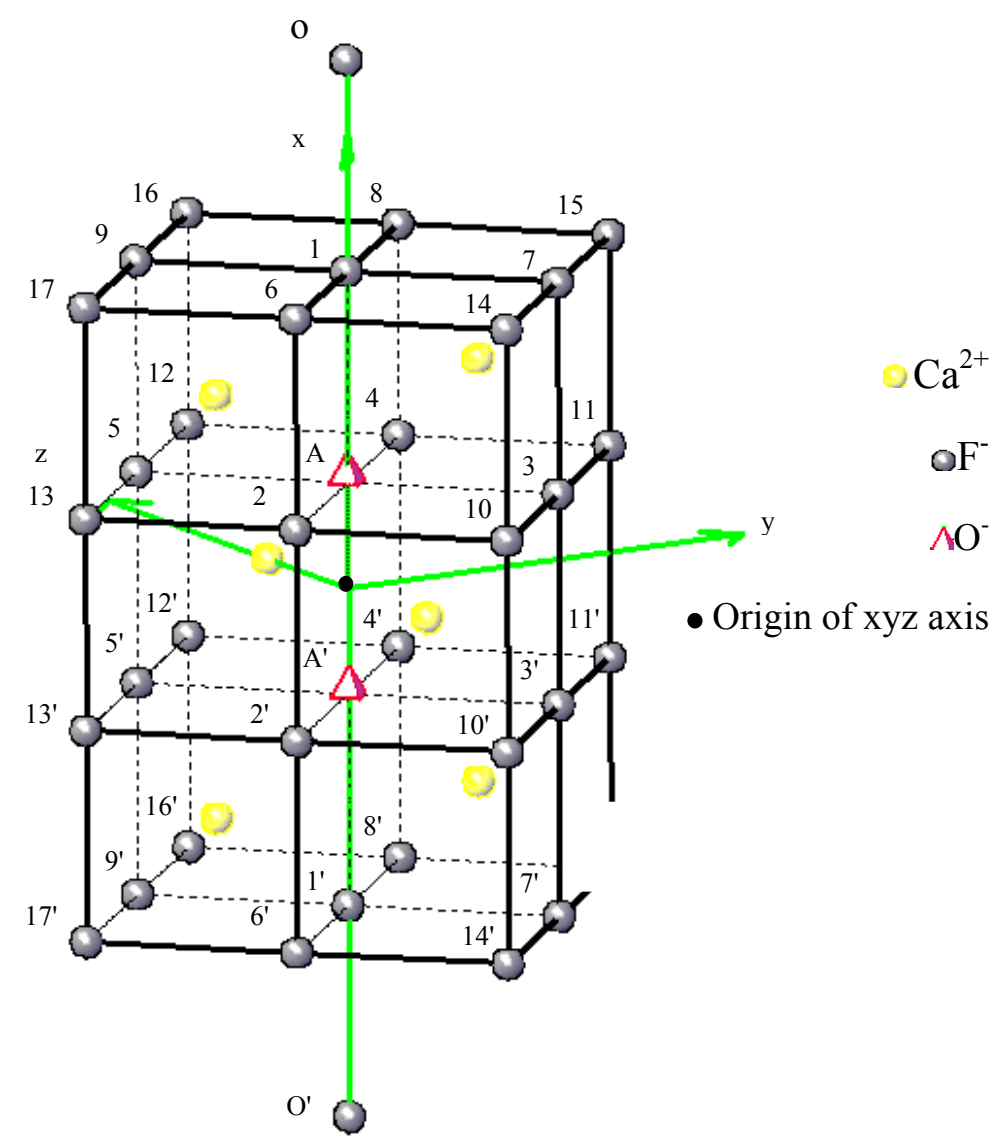

Fig.1: Theoretical model of the oxygen centepr. Ions which transform one into other are labeled the same but with prime.

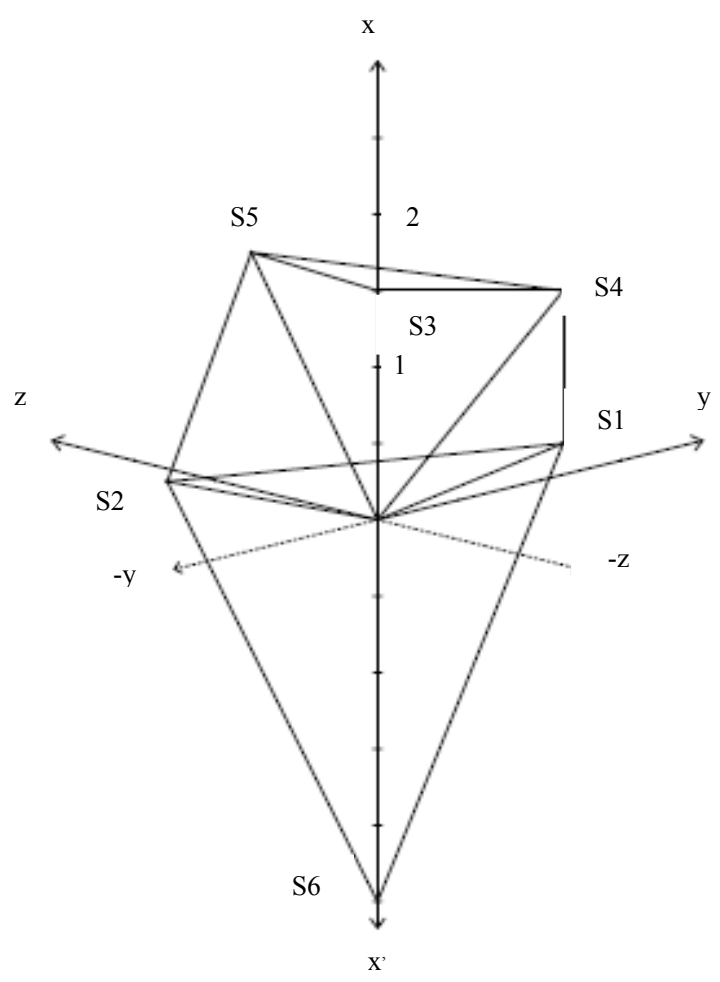

Fig. 2: The six different sites according to the coordinate system assumed in Fig. 1. 
When applying a magnetic field $\overline{\mathbf{B}}$ along definite directions, a detailed geometrical calculations have been done to relate the angles $(\phi, \Psi, \theta)$ done by the magnetic field $\overline{\mathbf{B}}$ and the $\left(x^{\prime}, y^{\prime}, z^{\prime}\right)$ coordinate system, for the four different combinations of axes, with the angle $\alpha$ designated between $\overline{\mathbf{B}}$ and the principal axis under consideration.

Using the physical model discussed earlier and the known alignment of the crystal with respect to $\overrightarrow{\mathbf{B}}$, it turns out that we expect four types of angular variation of the mean interaction frequency and four types of angular variation of the resultant displacement of interaction frequency as the magnetic field $\overrightarrow{\mathbf{B}}$ is rotated.

Table 1: Direction cosines $1, \mathrm{~m}, \mathrm{n}$ for the g-tensor $\mathrm{O}^{-}$for sites 1 to 6 .

\begin{tabular}{|c|c|c|c|c|c|c|}
\hline $\begin{array}{c}\text { Direction } \\
\text { cosines }\end{array}$ & Site 1 & Site 2 & Site 3 & Site 4 & Site 5 & Site 6 \\
\hline I & 0.4430 & 1 & 0.3333 & 0.8320 & 0.7261 & 1 \\
\hline m & 0.7071 & 0 & 0 & 0.7071 & 0.6860 & 0 \\
\hline n & 0.7071 & 0 & 0.9428 & -0.7071 & 0 & 0 \\
\hline
\end{tabular}

Table 2: Maximum frequency and the geometric parts of tensors for the six sites.

\begin{tabular}{|c|c|c|c|c|c|c|}
\hline $\mathbf{F}(\mathbf{M H z})$ & Site 1 & Site 2 & Site 3 & Site 4 & Site 5 & Site 6 \\
\hline $\mathbf{f}_{\mathbf{m a x}}$ & 2.6272 & 1.0876 & 1.0876 & 0.6263 & 0.4189 & 0.2349 \\
\hline $\mathbf{A}_{\mathbf{x x}}$ & -1.0509 & 2.1752 & -0.7251 & 0.6745 & 0.2464 & 0.4698 \\
\hline $\mathbf{A}_{\mathbf{x y}}$ & 2.2292 & 0 & 1.0254 & 0.6131 & 0.6259 & 0 \\
\hline $\mathbf{A}_{\mathbf{x z}}$ & 2.2292 & 0 & 0 & 0.6131 & 0 & 0 \\
\hline $\mathbf{A}_{\mathbf{y x}}$ & 2.2292 & 0 & 1.0254 & 0.6131 & 0.6259 & 0 \\
\hline $\mathbf{A}_{\mathbf{y y}}$ & 0.5254 & -1.0876 & 1.8127 & -0.3382 & 0.1725 & -0.2349 \\
\hline $\mathbf{A}_{\mathbf{y z}}$ & 2.9425 & 0 & 0 & 0.2890 & 0 & 0 \\
\hline $\mathbf{A}_{\mathbf{z x}}$ & 2.2292 & 0 & 0 & 0.6131 & 0 & 0 \\
\hline $\mathbf{A}_{\mathbf{z y}}$ & 2.9425 & 0 & 0 & 0.2890 & 0 & 0 \\
\hline $\mathbf{A}_{\mathbf{z z}}$ & 0.5254 & -1.0876 & -1.0876 & -0.3382 & -0.4189 & -0.2349 \\
\hline
\end{tabular}

Theoretical calculations were employed to evaluate the tabulated parameters to study the angular variation of mean interaction frequency obtained by the effects of the six sites and the angular variation of the resultant displacement of interaction frequency using the complete version of equation (6), when a constant applied magnetic field $\mathbf{B}=3340$ Gauss (resonance frequency for fluorine ion at $\mathbf{B}=3340$ Gauss is equal to $13.383 \mathrm{MHz}$ ), is rotated by an angle ranged from $0^{\circ}$ to $180^{\circ}$, for the following cases:

Case I: Rotation axis of $\overrightarrow{\mathbf{B}} / /[100]$ direction. The angular variation of mean interaction frequency and the angular variation of the resultant displacement of interaction frequency, for magnetic field orientations in the [100] plane of the crystal were analyzed for two cases correspond to two different combinations of coordination systems $\left(\mathbf{x}^{\prime \prime}, \mathbf{y}^{\prime}, \mathbf{z}\right)$ with the direction of $\overrightarrow{\mathbf{B}}$. These cases are illustrated in Fig. 3, 4, 5 and 6. 


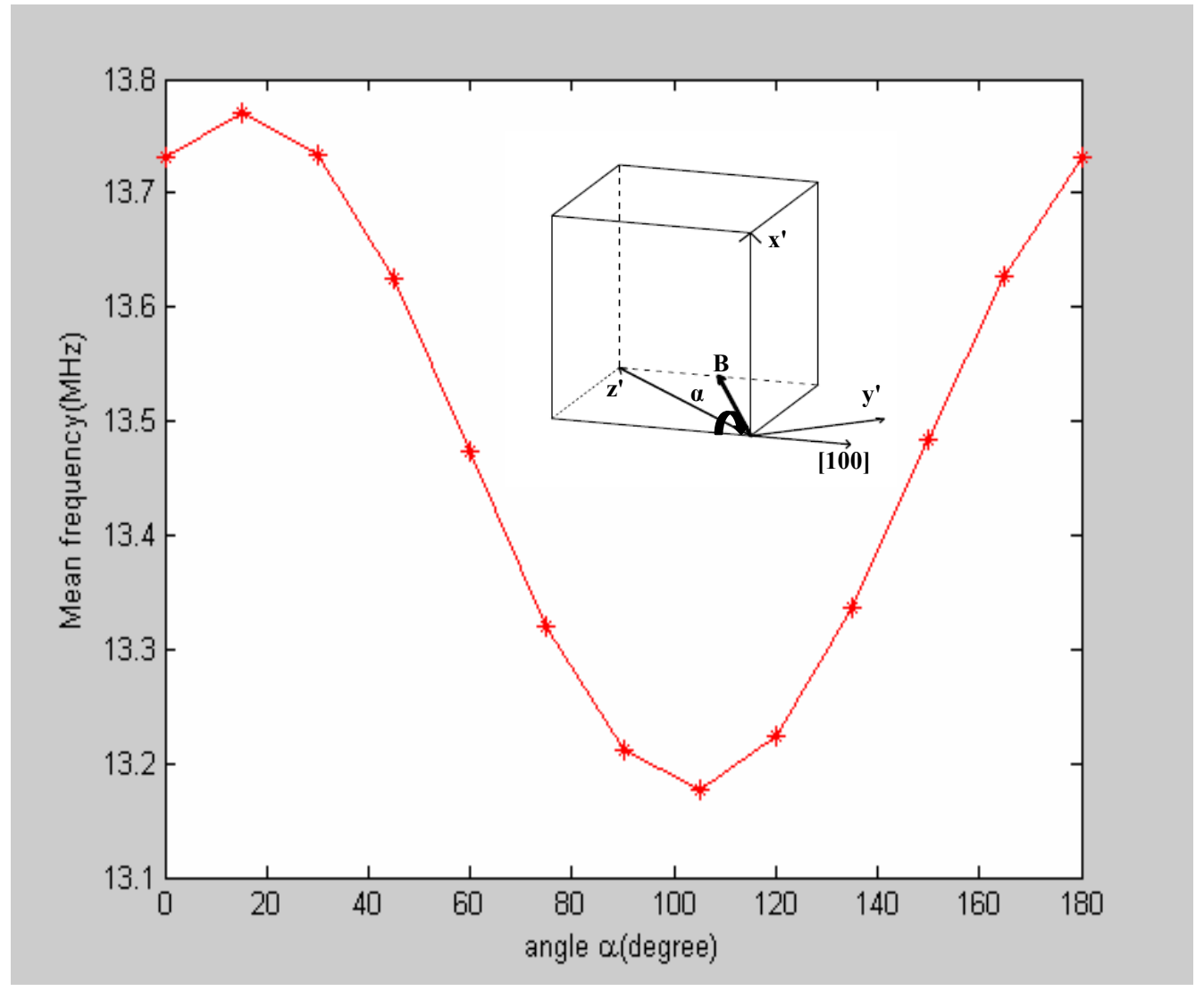

Fig. 3: Mean interaction frequency of six sites vs the direction of $\overrightarrow{\mathrm{B}}$. Rotation axis of $\bar{B} \backslash[100]$ : case 1

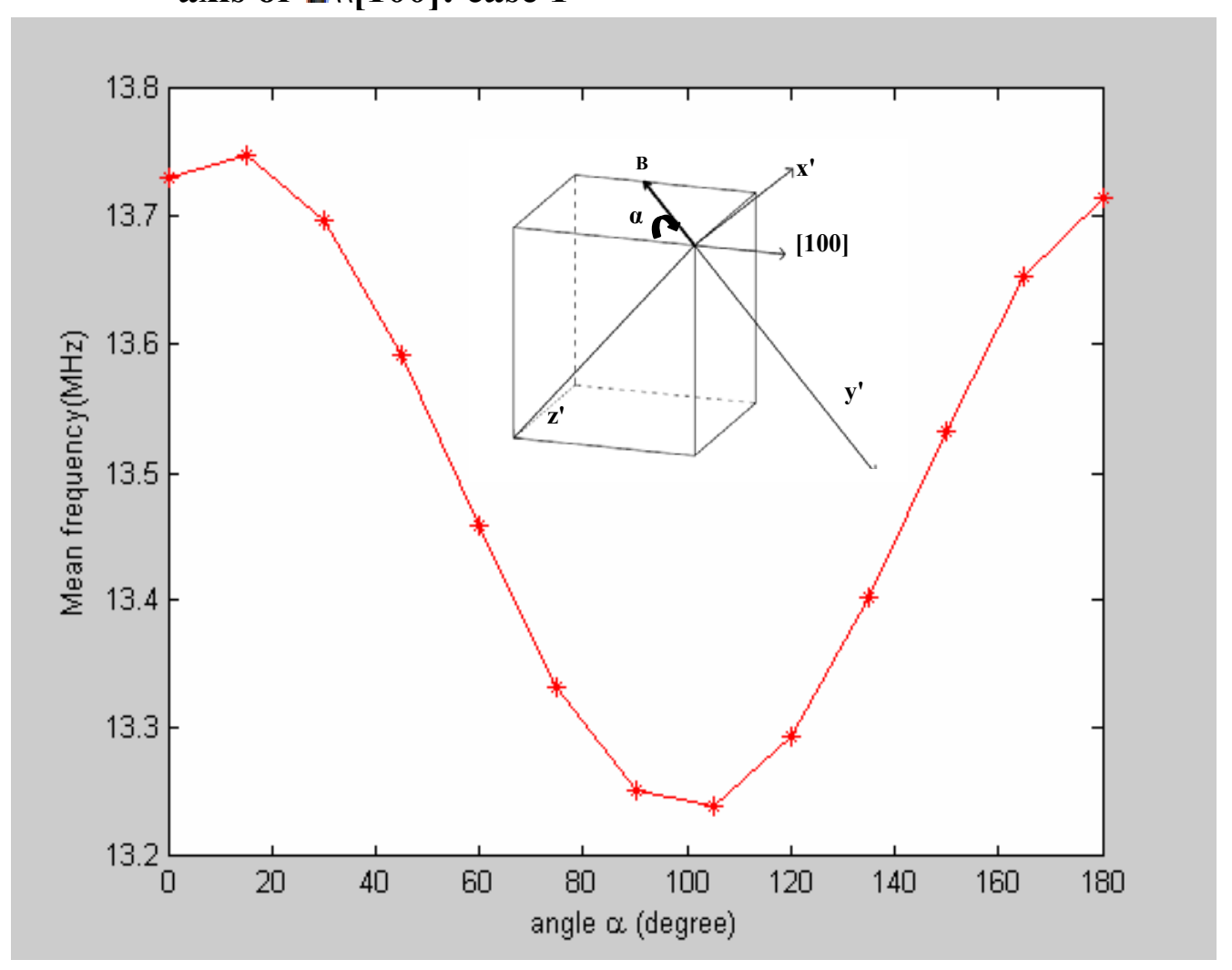

Fig. 4: Mean interaction frequency of six sites vs the direction of $\overline{\mathrm{B}}$. Rotation axis of $\vec{B} \backslash[100]$ : case 2 


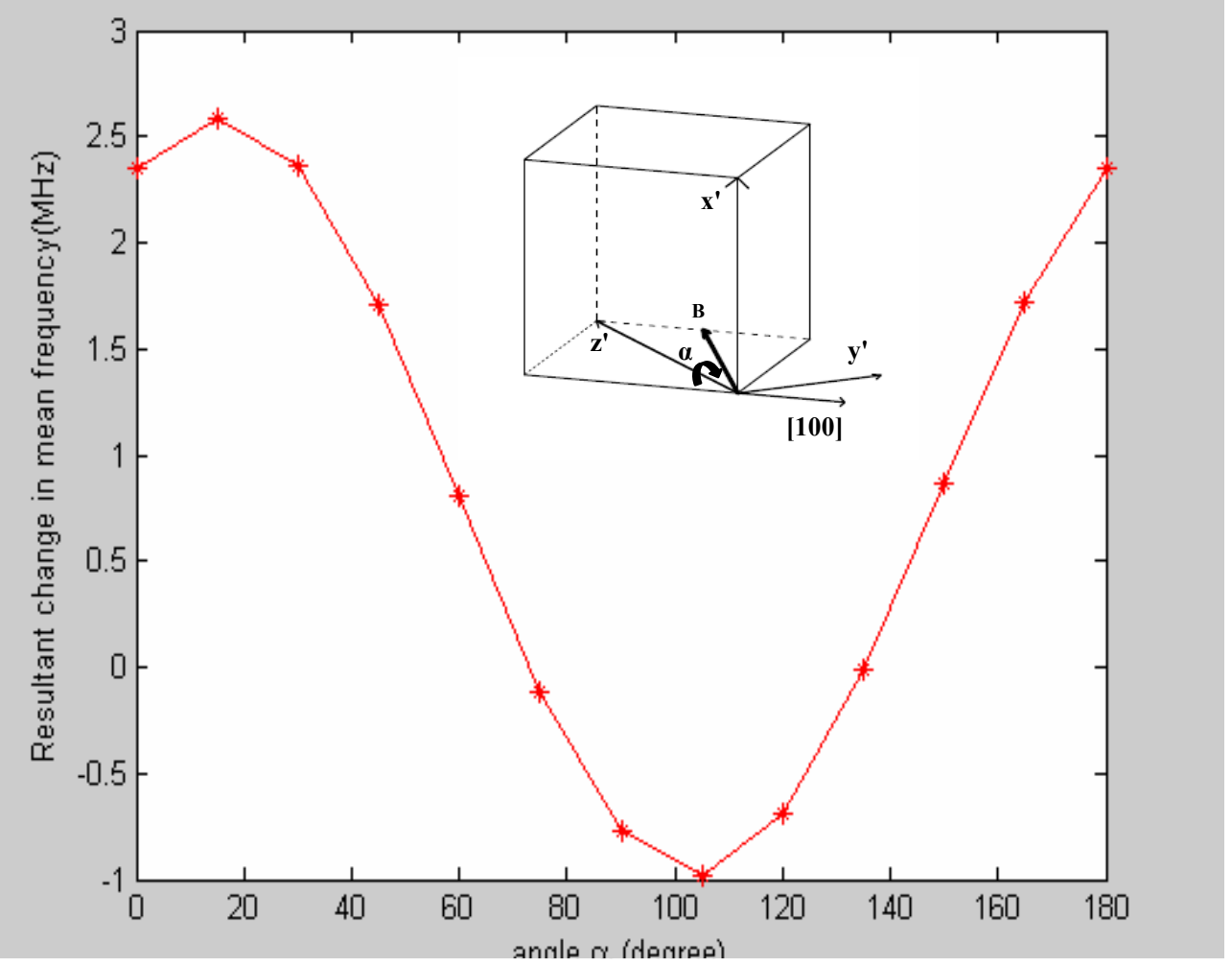

Fig. 5: Resultant change of mean frequency vs the direction of $\overline{\mathbf{B}}$. Rotation axis//[100]: case 3

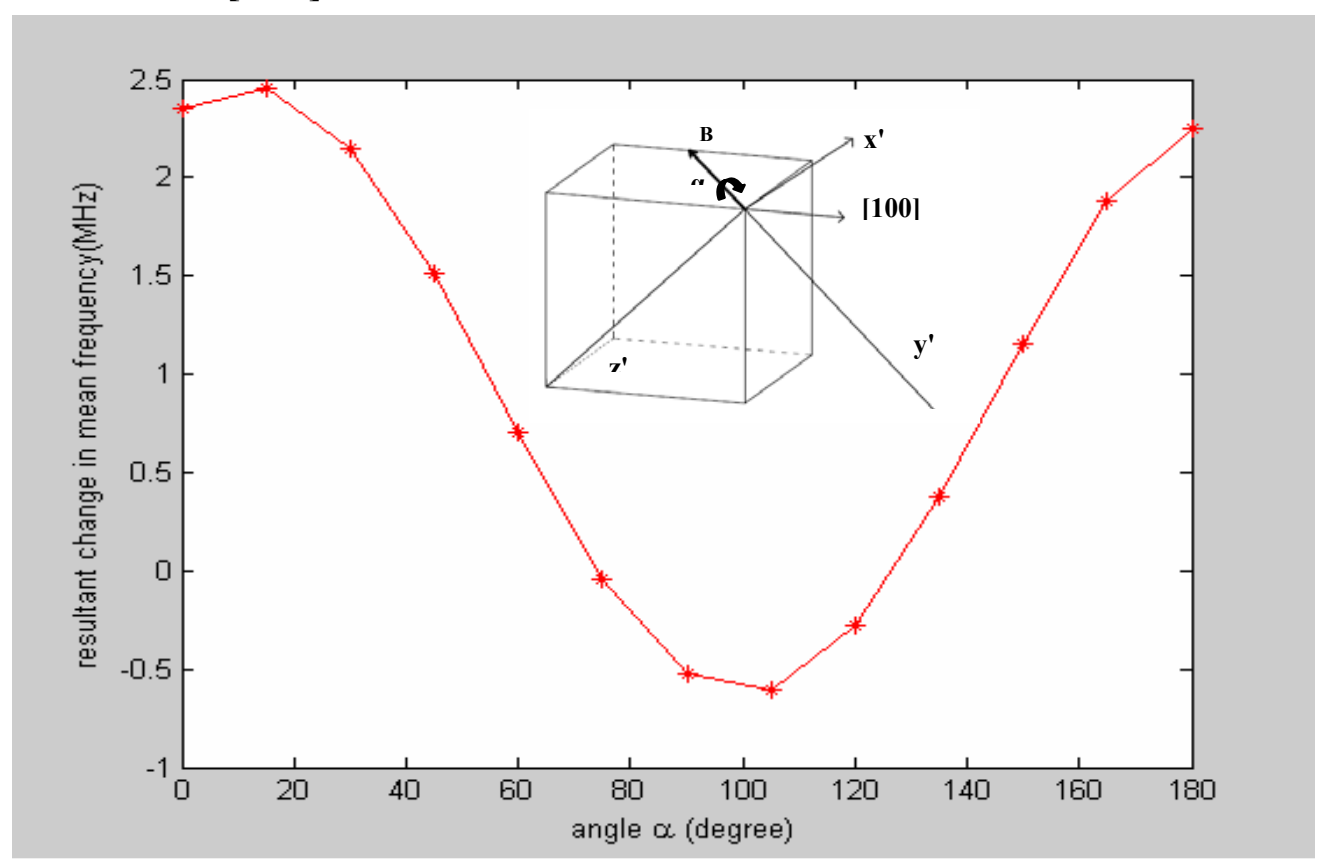

Fig. 6: Resultant change of mean frequency vs the direction of $\bar{B}$. Rotation axis//[100]: case 4 
Case II: Rotation axis of $\overline{\mathbf{B}} / /[110]$ direction. The angular variation of the interaction frequency and the angular variation of the resultant displacement of interaction frequency, for magnetic field orientations in the [110] plane of the crystal were again analyzed for another two cases correspond to the two different combinations systems of $(x, y, z)$ with the direction of $\overline{\mathbf{B}}$. These cases are illustrated in Fig. 7, 8, 9 and 10 .

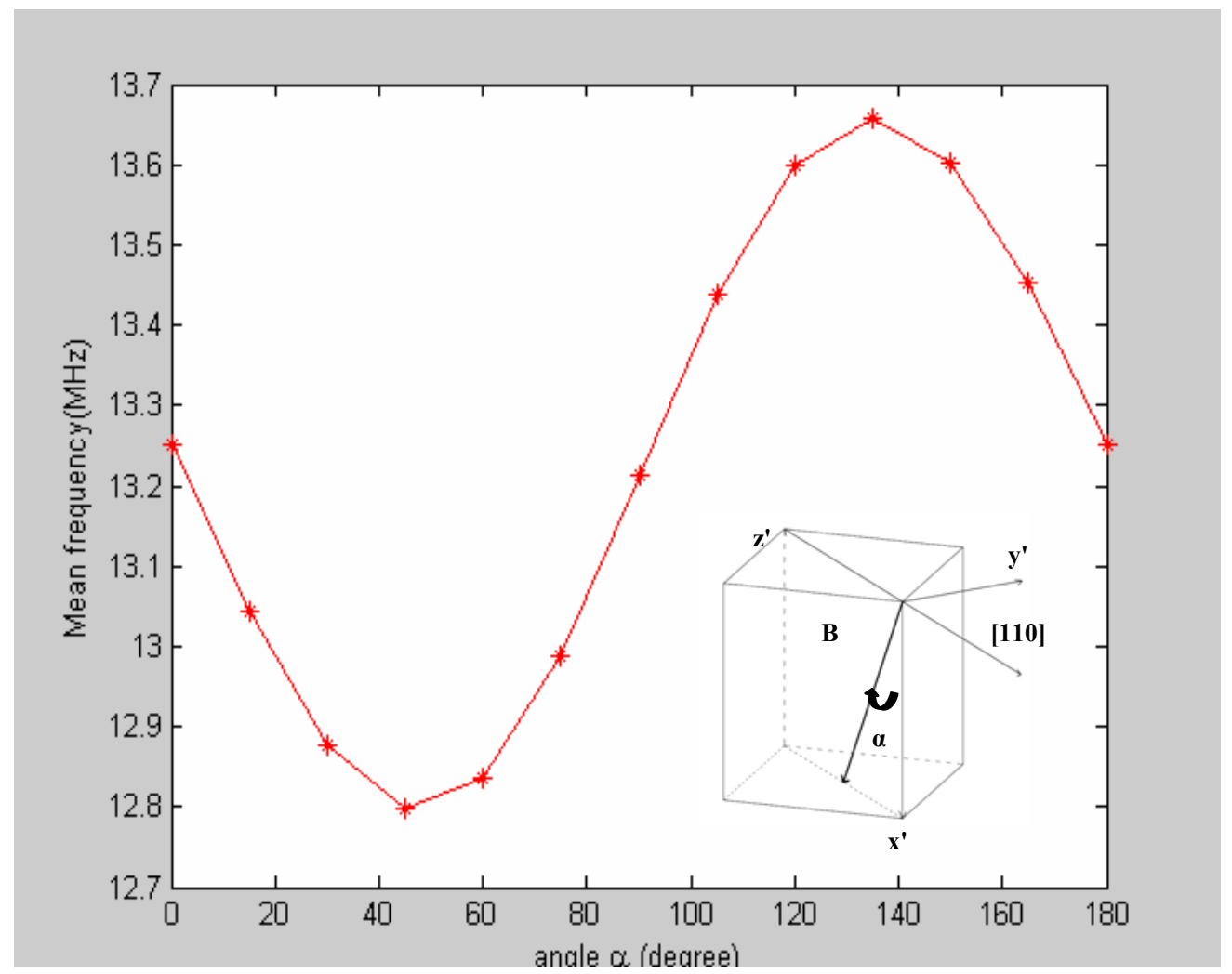

Fig. 7: Mean interaction frequency of six sites vs the direction of $\overline{\bar{B}}$. Rotation axis of $\vec{B} \backslash[110 \mid$ : case 1

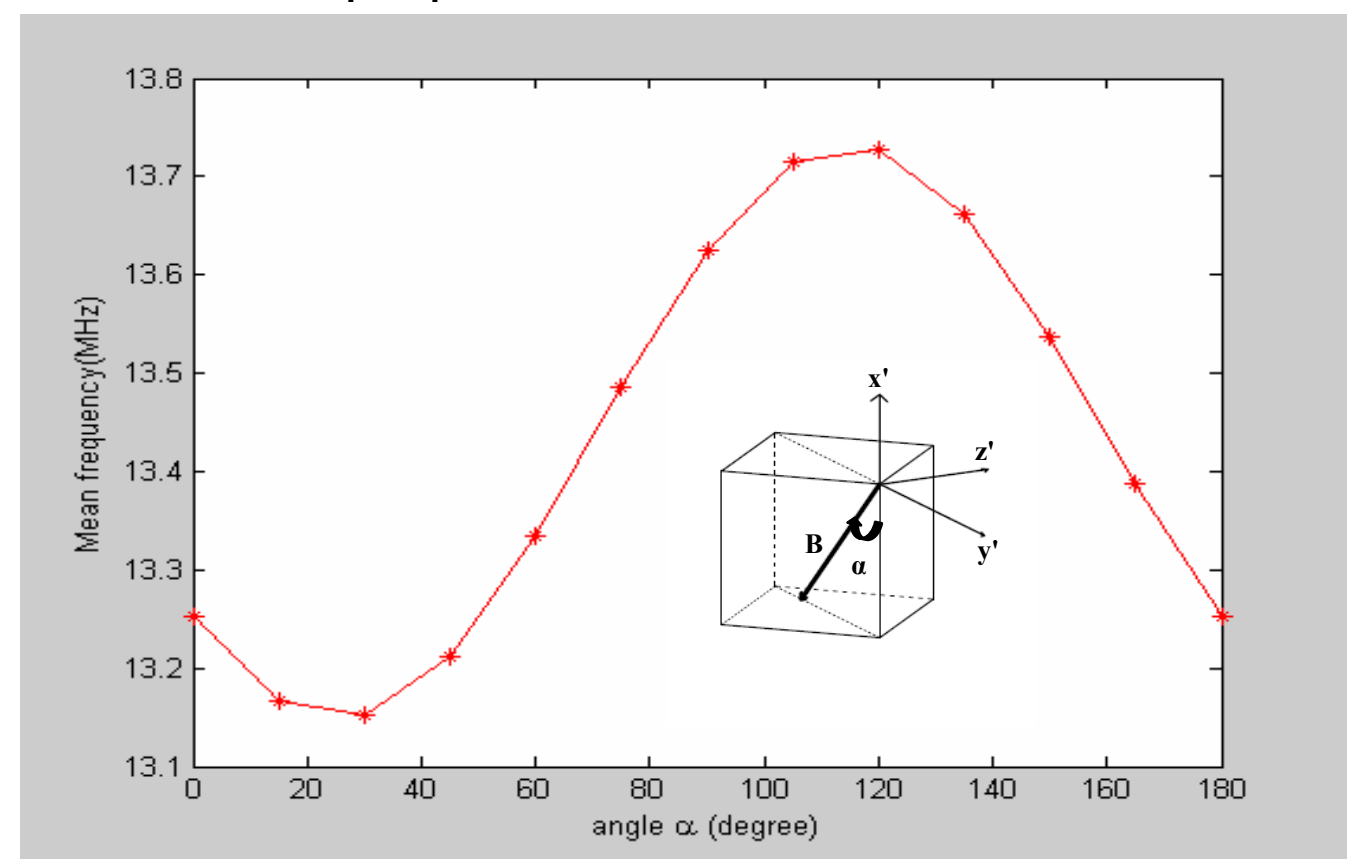

Fig. 8: Mean interaction frequency of six sites vs the direction of $\vec{B}$. Rotation axis of $\bar{B} \backslash[110]$ : case 2 


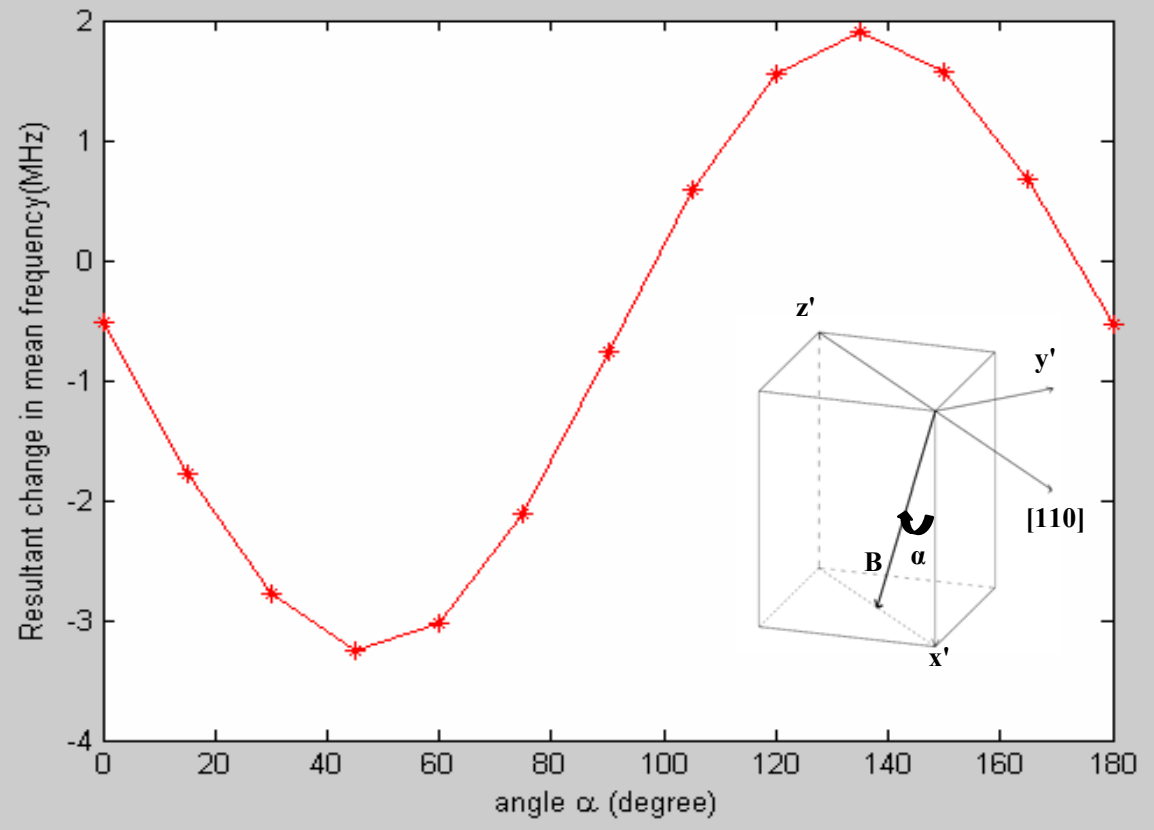

Fig. 9: Resultant change of mean frequency vs the direction of $\bar{B}$ Rotation axis//[110]: case 3

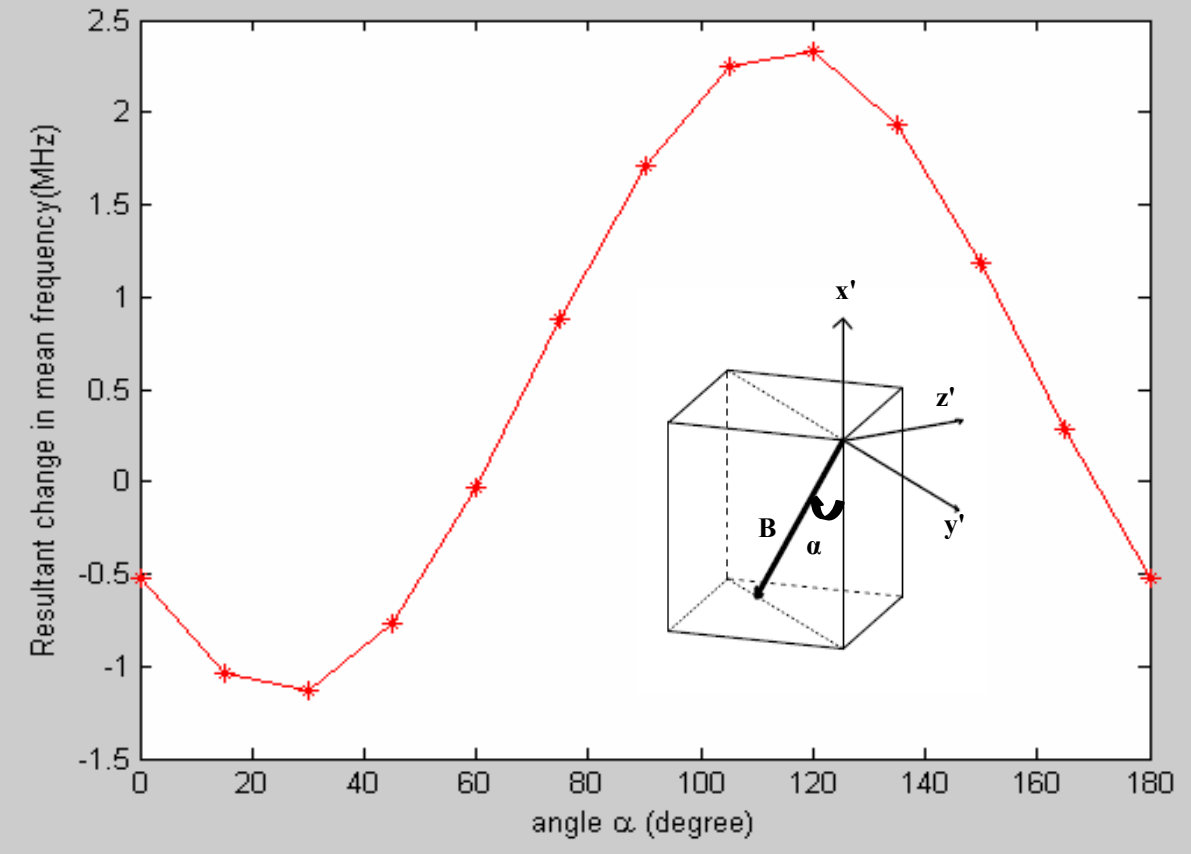

Fig. 10: Resultant change of mean frequency vs the direction of $\overline{\mathrm{B}}$. Rotation axis//[110]: case 4 


\section{CONCLUSION}

Theoretical and experimental studies of angular and magnetic fields effects of dipoledipole interaction frequencies, provide a means of extending the frequency range of available and convenient resonance centers in particular solid state laser active medium, such as oxygen centers in $\mathrm{CaF}_{2}$ crystals. These investigations marked the importance of intense studies of magnetic and geometric properties of the laser medium, i.e., considering the response of the paramagnetic material at the atomic level to the effects of strength and orientation of the applied external magnetic field. Tuning range of dipole-dipole interaction frequencies can be extended by various combinations of magnetic field strengths and geometrical orientations of active medium crystal.

\section{REFERENCES}

Abragam, A.; Bleaney, B. (1970). "Electron Paramagnetic Resonance of Transition Ions". Claredon Press, Oxford. 492 p.

Bill, H.; Silsbee, R.H. (1974). Dynamical jahn-teller and reorientation effects in the EPR spectrum of $\mathrm{CaF}_{2}: 0^{-}$. Phys. Review., 10(7) , 2697-2709.

Graybeal, J.D. (1988). "Molecular Spectroscopy". McGraw-Hill, Singapore. pp.218-221.

Kasir, F.A. (1994). Angular and magnetic field dependence of dipolar interaction frequency of $\mathrm{H}_{1}^{0}$ in $\mathrm{CaF}_{2}$ crystal. J. Ed. and Sci., 17, 167-185.

Megerle, U.; Pughesi, I.; Schriever, C.; Sailer, C.F.; Riedle, E. (2009). Sub-50 fs broadband absorption spectroscopy with tunable excitation: putting the analysis of ultrafast molecular dynamics on solid ground. Apply Phys. B: Laser Optics., 96, 215-231.

Mysovsky, A.S.; Radzhabov, E.A.; Reichling, M.; Shluger, A.L.; Sushko, P.V. (2005). Optical properties and transformation mechanism of oxygen centers and their aggregates in $\mathrm{CaF}_{2}$ crystals. Phys. Status Solidi., C2, 392-396.

Radzhbov, E.; Figura, P. (1986). Optical properties of oxygen-vacancy centers in fluorite. Phys. Status Solidi., B136, K55.

Shi, H.; Eglitis, R.I.; Borstel, G., (2007). Ab initio calculations of the oxygen-vacancy dipoles and $\mathrm{M}$ centers in $\mathrm{CaF}_{2}$. Computational Materials Science, 39, 430-436.

Sils, J.; Radzhbov, E.; Reichling, M. (2007). Characterization of oxygen defects in calcium difluoride. J. Phys. Chem. Solids., 68, 420-425. 